\title{
Resolving the stellar halos of six massive disk galaxies beyond the Local Group
}

\author{
Antonela Monachesi ${ }^{1}$, Eric F. Bell ${ }^{2}$, David J. Radburn-Smith ${ }^{3}$, Roelof \\ S. de Jong ${ }^{4}$, Jeremy Bailin ${ }^{5,6}$, Benne Holwerda ${ }^{7}$ and David Streich ${ }^{4}$ \\ ${ }^{1}$ Max Planck Institute for Astrophysics, Karl-Schwarzschild-Str. 1, Garching D-85748, \\ Germany \\ email: antonela@mpa-garching.mpg.de \\ ${ }^{2}$ Department of Astronomy, University of Michigan, 311 West Hall, 1085 South University \\ Ave., Ann Arbor, MI 48109, USA \\ ${ }^{3}$ Department of Astronomy, University of Washington, Seattle, WA 98195,USA \\ ${ }^{4}$ Leibniz-Institut für Astrophysik Potsdam, D-14482 Potsdam, Germany \\ ${ }^{5}$ Department of Physics and Astronomy, University of Alabama, Box 870324, Tuscaloosa, AL, \\ 35487, USA \\ ${ }^{6}$ National Radio Astronomy Observatory, P.O. Box 2, Green Bank, WV, 24944, USA \\ ${ }^{7}$ University of Leiden, Sterrenwacht Leiden, Niels Bohrweg 2, NL-2333 CA Leiden, The \\ Netherlands
}

\begin{abstract}
Models of galaxy formation in a hierarchical universe predict substantial scatter in the halo-to-halo stellar properties, owing to stochasticity in galaxies' merger histories. Currently, only few detailed observations of stellar halos are available, mainly for the Milky Way and M31. We present the stellar halo color/metallicity and density profiles of red giant branch stars out to $\sim 60 \mathrm{kpc}$ along the minor axis of six massive nearby Milky Way-like galaxies beyond the Local Group from the Galaxy Halos, Outer disks, Substructure, Thick disks and Star clusters (GHOSTS) HST survey. This enlargement of the sample of galaxies with observations of stellar halo properties is needed to understand the range of possible halo properties, i.e. not only the mean properties but also the halo-to-halo scatter, what a 'typical' halo looks like, and how similar the Milky Way halo is to other halos beyond the Local Group.
\end{abstract}

Keywords. galaxies: halos, galaxies: spiral, galaxies: individual (NGC 253, NGC 891, NGC 3031, NGC 4565, NGC 4945, NGC 7814), galaxies: photometry, galaxies: stellar content, galaxies: evolution

\section{Introduction}

Over the past decades different approaches have been used to observe stellar halos, a challenging task due to their faint surface brightnesses. Long exposure wide field imaging with photographic plates (Malin\&Carter 1980) and with small telescopes and wide field CCDs (e.g., Zheng et al. 1999, Martínez-Delgado et al. 2010) has allowed panoramic mapping of the brightest overdensities in nearby galaxies, revealing numerous tidal streams. Evidence of stellar halo substructures (e.g., stellar streams, shells, etc) in the outer regions of galaxies was possible with these types of images, proving the importance of merging in the galaxy formation process. However, none of these techniques allow for a detailed study of the physical properties of individual halos predicted by models, such as their age and metallicity as a function of galactocentric distance.

One of the best approaches for characterizing the properties of nearby galactic stellar halos is to study their resolved stellar populations. It is possible to measure stellar densities of resolved stars reaching equivalent surface brightnesses as faint as $\mu_{V} \sim 33$ mag $\operatorname{arcsec}^{-2}$, as well as measuring the stellar populations of those halos, which is crucial 

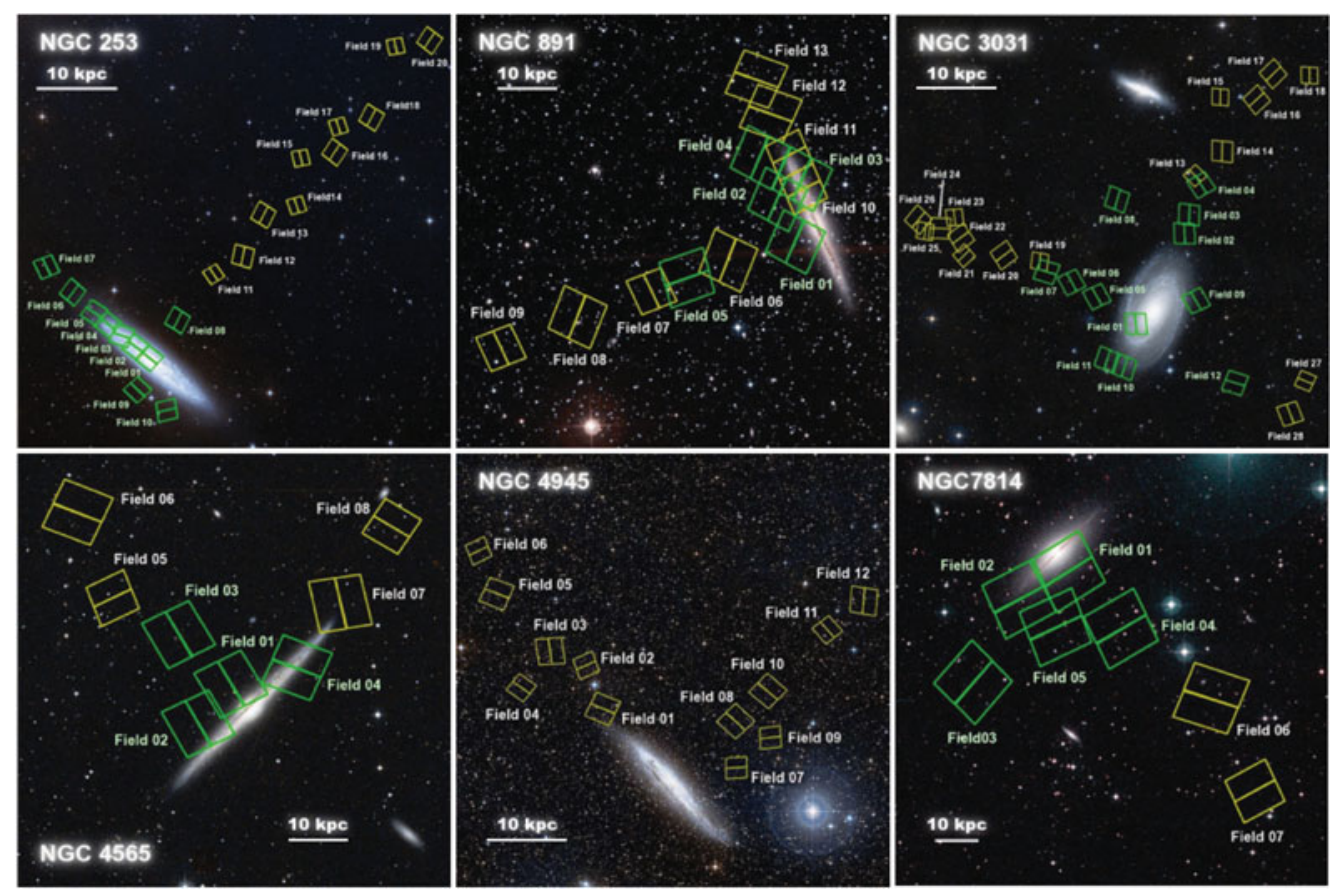

Figure 1. DSS colored images of the GHOSTS massive disk galaxies analyzed showing the locations of the HST ACS/WFC and WFC3/UVIS fields. North is up and east is to the left. ACS fields in green were introduced in R-S11 whereas ACS and WFC3 fields indicated in yellow are presented in Monachesi et al. (2016).

for testing model predictions (Monachesi et al. 2013). One such prediction is that there should be stellar population variations within a halo since the stellar population of halos should reflect the various satellites that form them. In particular, how a halo has formed and evolved is expected to leave strong imprint on its metallicity or abundance pattern (e.g., Font et al. 2006, Cooper et al. 2010, Gomez et al. 2012, Tissera et al. 2014).

The resolved stars of the Milky Way and M31 halos have been extensively studied. While both halos have similarities and qualitatively agree within the LCDM preferred paradigm of hierarchical formation, they exhibit clear differences. There is an order of magnitude difference in their stellar halo mass (e.g., Bell et al. 2008, Ibata et al. 2014) and a factor of 5 difference in metallicity and difference in gradient, which betray large differences in halo growth history (Deason et al. 2013, Gilbert et al. 2014). Cosmological models of galaxy formation predict that there should be large variations among the properties of individual halos in disk galaxies with similar mass (e.g., Bullock\&Johnston 2005, hereafter BJ05, Cooper et al. 2010, Tissera et al. 2014). Given the stochasticity involved in the process of galaxy formation, predictions such as differences in metallicity profiles, fraction of stellar halo created in-situ and accreted, stellar halo morphology, etc., need to be compared against observations to differentiate between the models and quantify the predicted halo-to-halo scatter. This motivates the study of other stellar halos, which was one of the main goals of the GHOSTS survey.

\section{The GHOSTS survey and sample of galaxies}

The GHOSTS survey (Galaxy Halos, Outer disks, Substructure, Thick disks, and Star clusters survey, Radburn-Smith et al. 2011, hereafter R-S11) is an extensive HST 
program dedicated to resolve the stars in the outskirts of 16 nearby disk galaxies of different masses, luminosities, and inclinations. Various fields along the principal axes of each galaxy were observed reaching projected distances as far as $\sim 70 \mathrm{kpc}$ from the galactic center. GHOSTS is the largest study of resolved stellar populations in the outer disk and halo of disk galaxies to date and its observations offer a direct test of model predictions. GHOSTS observations provide star counts and color-magnitude diagrams (CMDs) reaching typically 2-3 magnitudes below the tip of the red giant branch (TRGB). Using the RGB stars as tracers of the stellar halo population, we are able to study the size and shape of each stellar halo as well as the properties of their stellar populations such as age and metallicity.

In this work, we analyze the more massive disk Milky Way-like galaxies of our sample, namely NGC 253, NGC 891, NGC 3031, NGC 4565, NGC 4945, and NGC 7814. They have maximum rotation velocity $V_{\text {max }} \gtrsim 170 \mathrm{~km} / \mathrm{s}$ and their distances range from 3.5 to $15 \mathrm{Mpc}$. Two of these galaxies are edge-on and four are highly inclined. This facilitates studies of their halos, because we expect minimal to no disk contamination in projected distances beyond 5 or $10 \mathrm{kpc}$ when they are observed along their minor axis. Fig. 1 shows the field locations of the GHOSTS galaxies studied here.

\section{Results: Stellar halo density and color profiles}

The data reduction steps and photometry were performed using the GHOSTS pipeline described in R-S11 and Monachesi et al. (2015). Stellar photometry on the images was performed using the ACS and WFC3 modules of DOLPHOT, a modified version of HSTPHOT (Dolphin 2000). The final output of DOLPHOT provides instrumental VEGA magnitudes, already corrected for CTE loss and with aperture corrections calculated using isolated stars. We show in Fig. 2 the CMDs of two fields, from NGC 3031 and NGC 253. The CMDs are dereddened for Galactic extinction and are free of contaminants. The unresolved background galaxies (the major source of contamination in our observations) were eliminated using photometric 'culls' determined from diagnostic parameters included in the photometric output of DOLPHOT. We can clearly see halo detections in the outer fields observed, with projected distances between 50 and $70 \mathrm{kpc}$ from the galactic center. In general the CMDs of the fields shown in Fig. 1 are mostly populated by old RGB stars (older than 1 Gyr). There are however younger populations such as blue, extended main sequence stars $(<500 \mathrm{Myr})$ or massive stars burning helium in their core (25-600 Myr old red and blue loop sequence stars). These appear primarily in the fields closer than $R \sim 15 \mathrm{kpc}$ to each galaxy, and especially along the major axis, which are dominated by disk stars.

The stellar density profiles and color profiles of two of the galaxies analyzed are also shown in Fig 2 for NGC 3031 (left panels) and NGC 253 (right panels). The results for all galaxies and details on how these results were obtained can be found in Monachesi et al. (2015) and Harmsen et al. (in prep.). Briefly, we selected RGB stars within a magnitude range that ensures all of the stars to be in a CMD region with completeness higher than $70 \%$. This is typically from the TRGB down to $\sim 0.7$ mag below it. We measured the projected stellar density in each of the fields observed and constructed their stellar density profiles (middle panels in Fig. 2). The equivalent $V$-band surface brightness values are indicated on the right hand $y$-axis. Errorbars indicate Poisson uncertainty. We fit power-law functions to the projected minor axis density profiles over 10 to $70 \mathrm{kpc}$ and find slopes between $-2.2>\alpha>-4$ for all our galaxies. Deviations from the power law fits that cannot be accounted for from Poisson uncertainty most 
likely indicate halo substructure. The scatter around the power law profiles is $\sim 0.18$ dex, which may suggest that there is abundant substructure (RMS/total $\sim 40 \%$ ).

The minor axis stellar halo color profiles (bottom panels in Fig. 2) were obtained from the median $F 606 W-F 814 W$ colors of the selected RGB stars in each field as a function of projected radius. In order to have a clean stellar halo profile and to avoid the disk as much as possible we do not use the major axis fields to construct these profiles. Error bars indicate uncertainties in the median values calculated by bootstrapping our sample of stars as well as systematic uncertainties due to calibration which accounts for up to $\sim 0.04 \mathrm{mag}$ in colors. The red line in the color profiles shows a linear fit to the data. We find that half of the galaxies (NGC 4565, NGC 0891, and NGC 7814) show fits consistent with stellar halo color gradients whereas the remaining three galaxies (NGC 0253, NGC 3031, and NGC 4945) have rather flat color profiles. Because the colors of the RGB stars are more sensitive to metallicity than to age and because there is a direct relation between RGB colors and metallicities (Streich et al. 2014), one can assume that the color profiles reflect metallicity profiles. The right hand $y$-axes indicate the $[\mathrm{Fe} / \mathrm{H}]$ values that the colors correspond to, calculated from the relation derived by Streich et al. (2014) and assuming $[\alpha / \mathrm{Fe}]=0.3$. The metallicities $[\mathrm{Fe} / \mathrm{H}]$ will be lower or higher for a given color in case of $[\alpha / \mathrm{Fe}]$ larger or lower than 0.3 , respectively.

In Figure 3 we show the stellar halo color profiles of all the galaxies together, where we can see the diversity in the color profiles of massive disk galaxies. Since the galaxies studied in this work are all Milky Way-like galaxies, we find that there is a wide range in stellar halo colors and a diversity of color/metallicity profiles for galaxies of similar mass and luminosity. The black dashed line in this figure represents the average color profile of the 11 BJ05 stellar halo models, which were analyzed in the exact same way as the data (see Monachesi et al. 2013) and the shaded area represents the 1- $\sigma$ model-to-model scatter from the average.

\section{Conclusions and future work}

We analyze the halo stellar populations of six massive highly inclined disk Milky Waylike galaxies from the GHOSTS survey. Several fields along the principal axes of each galaxies were observed from which we constructed CMDs showing halo populations out to projected distances between $\sim 50-70 \mathrm{kpc}$ along the minor axis. It is important to emphasize that all of these galaxies have halo stars out to at least $50 \mathrm{kpc}$ along the minor axis, which is more than 50 scale heights of the Milky Way's thick disk. Thus, our observations show that highly inclined massive disk galaxies $\left(V_{\text {max }} \gtrsim 170 \mathrm{~km} / \mathrm{s}\right)$ have clear extended stellar halos beyond the region where the disk dominates.

We use RGB stars along the minor axis of these galaxies as halo tracers and find that: 1) Half of the galaxies (NGC 0891, NGC 4565, NGC 7814) display a negative gradient in their color profiles, with bluer color in the outer regions. Three galaxies show flat color halo profiles (NGC 0253, NGC 3031, NGC 4945) reflecting negligible halo population variations as a function of galactocentric distances. 2) The projected minor axis stellar density profiles have power law slopes of $-4<\alpha<-2$ over 10 to $70 \mathrm{kpc}$ and significant scatter around the power-law fit of $\sim 0.18$ dex, which likely suggests the existence of abundant substructure (RMS/total $\sim 40 \%$ ).

The next step will be to quantitative compare our results against high resolution cosmological hydrodynamical simulations, such as EAGLE, (Schaye et al. 2015) that will help us to interpret the observations. We will generate simulated maps of RGB stars and analyze their properties in the exact same way as we did with the observations, highlighting areas of agreement with the data and areas where future improvement is required. 

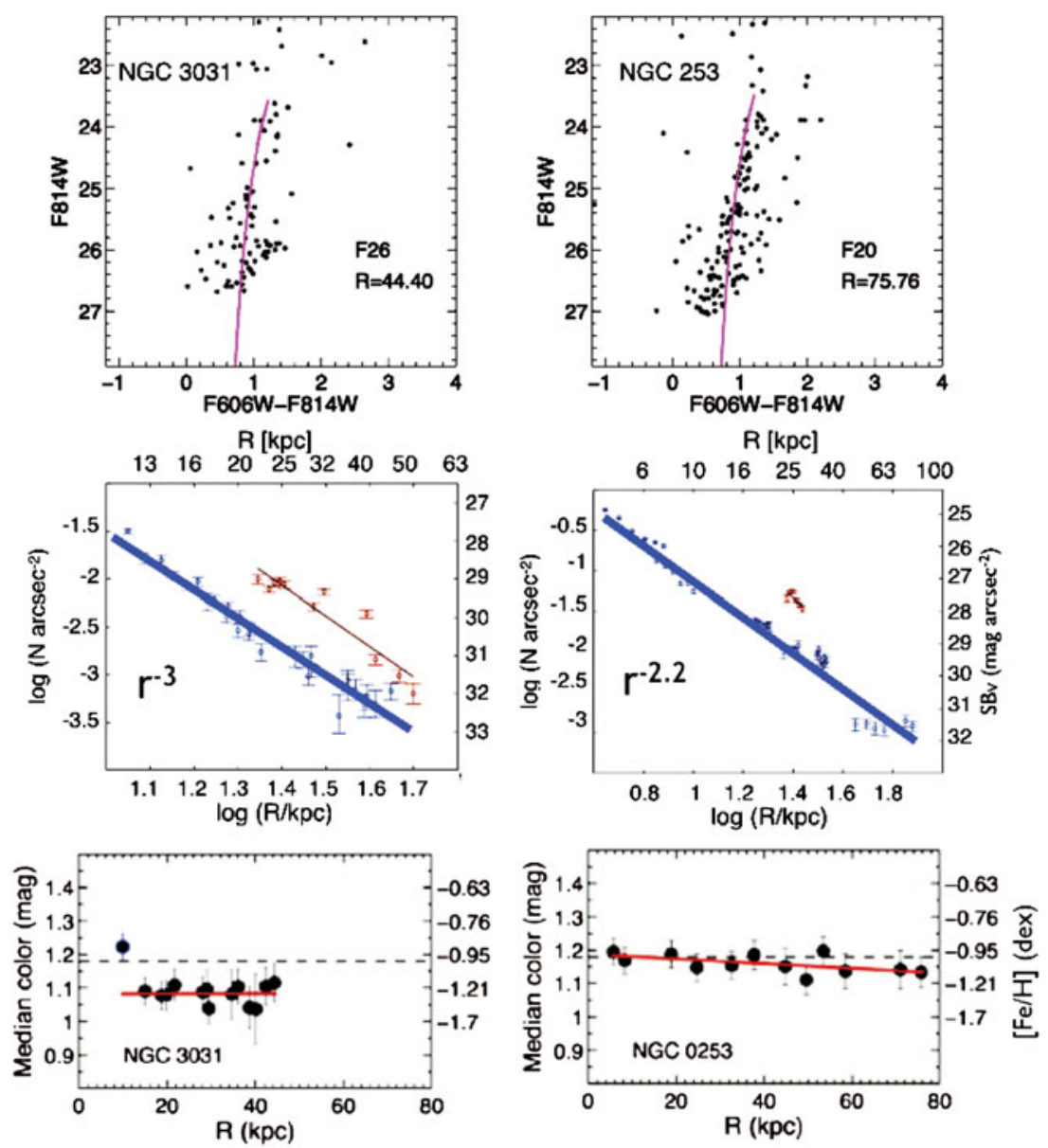

Figure 2. An example of our results for two of the GHOSTS galaxies analyzed: NGC 3031 (left panels) and NGC 253 (right panels). Results for all galaxies are presented in Monachesi et al. (2016) and Harmsen et al. (in prep.). Top panels: Color magnitude diagrams of stars, after unresolved background galaxies and other spurious detections have been removed, for two of the most distant fields. There are clear detections of RGB halo stars out to distances as far as $\sim 70 \mathrm{kpc}$ from the galactic center. Middle panels: Stellar density profiles along the minor axis (blue dots) and major axis (red dots) measured using the RGB stars between the TRGB and $0.7 \mathrm{mag}$ below it. We fitted the minor axis stellar density profiles with power-law functions and find slopes between $-4<\alpha<-2$ for all the GHOSTS massive galaxies. Deviations from these fits may indicate the presence of substructure in these halos. The equivalent $V$-band surface brightness is labeled on the right hand $y$-axis. We reach down to $\sim 32 \mu_{V}$. Bottom panels: Minor axis median color profiles obtained using the RGB stars between the TRGB and 0.7 mag below. The dashed line represents the average color profile obtained using the 11 BJ05 realizations. Metallicities have been estimated using the relation derived in Streich et al. (2014) and are indicated on the right hand $y$-axis. These two galaxies have flat minor axis metallicity profiles.

Finally, we note that our results are obtained from pencil-beam HST observations which, even though we have several fields per galaxy such that our results are representative of a relatively large portion of the stellar halo, do not map the entire halos of these galaxies. It is therefore important to combine our results with panoramic views of these galaxies, such as those that are being obtained with the PISCeS survey (see Crnojević's contribution in this proceeding) and Hyper Suprime-Cam (Okamoto et al. 2015) in order to fully understand the assembling history of these galaxies. 


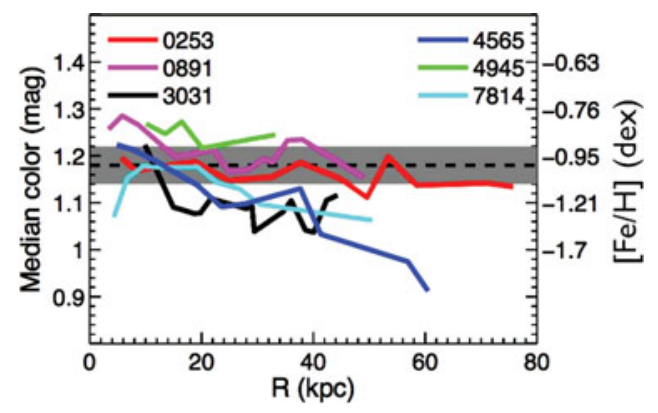

Figure 3. Minor axis color profiles of the stellar halos of the GHOSTS massive disk galaxies. Half of the galaxies display a negative gradient, with bluer colors in the outer regions. Three galaxies however show flat color halo profiles. The estimated metallicities are indicated on the right hand $y$-axis. The black dashed line indicates the average color profile of the 11 BJ05 stellar halo model realizations and the shaded area represents the 1- $\sigma$ model-to-model scatter from the average.

\section{References}

Bell, E. F., Zucker, D. B., \& Belokurov, V. et al. 2008, ApJ, 680, 295

Bullock, J. S. \& Johnston, K. V. 2005, ApJ, 635, 931 (BJ05)

Cooper, A. P., Cole, S., \& Frenk, C. S. et al. 2010, MNRAS, 406, 744

Deason, A. J., Belokurov, V., Evans, N. W., \& Johnston, K. V. 2013, ApJ, 763, 113

Dolphin, A. E. 2000, PASP, 112, 1383

Font, A. S., Johnston, Kathryn V., Bullock, J. S., \& Robertson, B. E. 2006, ApJ, 646, 886

Gilbert, K. M., Kalirai, J. S., \& Guhathakurta, P. et al. 2014, ApJ, 796, 76

Gomez, F. A., Coleman-Smith, C. E., \& O'Shea, B. W. et al. 2012, ApJ, 760, 112

Ibata, R. A., Lewis, G. F., \& McConnachie, A. W. et al. 2014, ApJ, 780, 128

Malin, D. F. \& Carter, D. 1980, Nature, 285, 643

Martínez-Delgado, D., Gabany, R. J., \& Crawford, K. et al. 2010, ApJ, 140, 962

Monachesi, A., Bell, E. F., \& Radburn-Smith, D. J. et al. 2013, ApJ, 766, 106

Monachesi, A., Bell, E. F., \& Radburn-Smith, D. J. et al. 2016, MNRAS, 457, 1419

Okamoto, S., Arimoto, N., \& Ferguson, A. M. N. et al. 2015, ApJL,809, 10

Radburn-Smith, D. J., de Jong, R. S., \& Seth, A. C. et al. 2011, ApJS, 195, 18(R-S11)

Schaye, J., Crain, R. A., \& Bower, R. G. et al. 2015, MNRAS, 446, 521

Streich, D., de Jong, R. S., \& Bailin, J. et al. 2014, A\&A, 563, A5

Tissera, P. B., Beers, T. C., Carollo, D., \& Scannapieco, C. 2014, MNRAS, 439, 3128

Zheng, Z., Shang, Z., \& Su, H. et al. 1999, AJ, 117, 2757 